\title{
钴掺杂氧化铈纳米粒子电催化性能研究
}

\author{
杨志宾 ${ }^{1}$ ，岳䑣联 ${ }^{1}$ ，余向南 ${ }^{1}$, 吴苗苗 ${ }^{2}$ \\ (中国矿业大学(北京) 1. 煤气化燃料电池中心; 2. 材料科学与工程学院, 北京 100083)
}

摘 要: 氧化铈的电子导电性较低、氧空位数量少, 难以单独用作为电催化剂。但是掺杂过渡金属或非金属元素可 以提高氧化铈的 $\mathrm{CO}$ 催化能力, 同时在氧化物中掺杂钴可有效提高材料的电催化能力, 因此本工作开展了对钴掺杂 的氧化铈电催化性能的研究。采用均相沉淀法制备了钴掺杂的氧化铈纳米粒子, 电化学测试发现当钴掺杂比例为 $20 \mathrm{~mol} \%$ 时, 氧化铈纳米粒子对氧气还原反应(ORR)和氧气析出反应(OER)的综合催化能力最强。经过 $10 \mathrm{~h}$ 的长时间 催化作用，ORR、OER 过程中的电流密度分别下降了 $20 \%$ 、5\%左右，远优于贵金属和未掺杂氧化铈纳米粒子催化 剂, 显示出良好的催化稳定性。拉曼光谱、阻抗图及 XPS 谱图等的测试分析表明钴掺杂后材料的电荷转移阻抗降 低(电子导电性的提高)、氧活性物种和氧空位增加是氧化铈催化性能提高的主要原因。本工作通过钴掺杂大幅度提 高了氧化铈的电催化性能，同时为其它离子导体作为双功能电催化剂的使用提供了借鉴。

关 键 词: 氧化铈; 掺杂; 电催化剂

中图分类号: TQ174 文献标识码: A

\section{Electrocatalytic Activity of Cobalt Doped Ceria Nanoparticles}

\author{
YANG Zhi-Bin ${ }^{1}$, YUE Tong-Lian ${ }^{1}$, YU Xiang-Nan ${ }^{1}$, WU Miao-Miao ${ }^{2}$
}

(1. Union Research Center of Fuel Cell, China University of Mining and Technology (Beijing), Beijing 100083, China; 2. Department of Materials Science and Engineering, China University of Mining and Technology (Beijing), Beijing 100083, China)

\begin{abstract}
It is very difficult for single ceria to be used as an electrocatalyst because of its relatively poor electron conductivity and rare number of oxygen vacancy. Recently, it has been studied in the field of $\mathrm{CO}$ catalysis by doping transition metallic or non-metallic elements to improve the catalytic ability of ceria, while recent research has demonstrated that many oxides containing cobalt display better electrocatalytic activity. In this study cobalt doped ceria nanoparticles were prepared by homogeneous precipitation method. The electrochemical tests show that the optimum doping molar ratio is $20 \mathrm{~mol} \%$ for ORR and OER catalytic effect. After 10 hours of catalysis, the current density of ORR and OER decrease by about $20 \%$ and $5 \%$, respectively, far below the corresponding values when noble metal and undoped cerium oxide nanoparticles were used as catalysts, and it indicated that the prepared catalyst owns good catalytic stability. In addition, XPS and other tests show that the decrease of charge transfer impedance (the improvement of electronic conductivity), the increase of active oxygen species, and the increased oxygen vacancies after doping are main reasons for improved catalytic performance. Therefore, doping cobalt greatly enhanced electrocatalytic properties of ceria nanoparticles are greatly enhanced by doping cobalt, providing guidence for other ionic conductors employed as bifunctional electrocatalysts.
\end{abstract}

Key words: ceria oxide; doping; electrocatalyst 
随着化石能源逐渐枯竭以及新能源行业的不断 壮大, 电化学能源器件的应用越来越普及。其中, 金 属-空气电池由于具有能量密度较高和环境友好的 突出优点而受到了广泛关注。然而此类电池的阴极 存在氧气还原反应 $(O R R)$ 和氧气析出反应 $(O E R)$ 动 力学缓慢的问题 ${ }^{[1-2]}$, 目前公认最有效的 ORR 和 OER 催化剂分别是 $\mathrm{Pt}$ 基催化剂和 $\mathrm{RuO}_{2}$ 基催化剂。 但是 $\mathrm{Pt}, \mathrm{Ru}$ 资源稀缺、价格昂贵，且 $\mathrm{Pt}$ 基和 $\mathrm{RuO}_{2}$ 基催化剂循环稳定性不高, 而难以大规模商业化应 用。因而开发高效, 稳定的非贵金属催化剂替代 $\mathrm{Pt}$ 基和 $\mathrm{RuO}_{2}$ 基催化剂成为了研究热点 ${ }^{[3-5]}$ 。

氧化铈由于结构简单、铈元素含量丰富(地壳中 的含量甚至超过渡金属铜、锡)和催化性能优良等原 因, 受到研究者的广泛重视, 早已应用在众多催化 领域。但在常温下, 单独的氧化铈有电子导电性较 差等缺点, 导致其电催化性能不佳, 难以作为金属空气电池中的阴极催化剂来使用, 必须通过改性来 提高其对 ORR 和 OER 的电催化能力。已有的研究 表明过渡金属元素或稀土元素掺杂氧化铈能够提高 其对 $\mathrm{CO}$ 催化 ${ }^{[6-7]}$ 和光催化 ${ }^{[8]}$ 等能力。而掺杂是否影 响氧化铈在常温下对 ORR 和 OER 的电催化性能以 及产生影响的机理却没有相关报道, 因此本文以过 渡金属钴作为异元素来掺杂氧化铈, 研究掺杂是否 会改变氧化铈对 ORR 和 OER 的电催化能力以及造 成性能变化的原因。

为了避免掺杂影响形貌从而改变催化性能, 本 研究采用均相沉淀法制备了不同钴含量掺杂的氧化 铈纳米粒子(零维材料, 不影响掺杂), 采用旋转环 盘电极测试钴掺杂氧化铈在碱性介质中的电催化能 力, 并分析钴掺杂后氧化铈对 ORR 和 OER 电催化 能力提高的原因, 同时为其它离子导体作为金属空气电池中双功能催化剂提供借鉴作用。

\section{1 实验方法}

\section{1 试剂}

实验采用试剂主要包括: 六水合硝酸铈 $\left(\mathrm{Ce}\left(\mathrm{NO}_{3}\right)_{3} \cdot 6 \mathrm{H}_{2} \mathrm{O}, \mathrm{AR}, 99.0 \%\right)$ 、六水合硝酸钴 $\left(\mathrm{Co}\left(\mathrm{NO}_{3}\right)_{3} \cdot 6 \mathrm{H}_{2} \mathrm{O}, \mathrm{AR}, 99.0 \%\right)$ 、双氧水 $\left(\mathrm{H}_{2} \mathrm{O}_{2}\right.$, $30 \mathrm{wt} \%$ )、氨水 $\left(\mathrm{NH}_{3} \cdot \mathrm{H}_{2} \mathrm{O}, 25 \sim 28 \mathrm{wt} \%\right.$ ) 和乙炔黑 (电池 级) 购于国药化学集团有限公司; 无水乙醇 $\left(\mathrm{CH}_{3} \mathrm{CH}_{2} \mathrm{OH}, \mathrm{AR}, 99.9 \%\right.$, 江苏强盛功能化学股份有 限公司); Nafion 溶液(5wt\%, Dupont D520, 阿法埃 莎(中国)化学有限公司 ); $\mathrm{Pt} / \mathrm{C}$ 催化剂(Pt 20wt\%, 阿 法埃莎(中国)化学有限公司); $\mathrm{RuO}_{2}$ 催化剂 $(99.9 \mathrm{wt} \%$, 阿法埃莎(中国)化学有限公司)。

\section{2 催化剂的制备}

实验采用均相沉淀法制备掺杂钴的氧化铈纳米 粒子。首先, 将不同摩尔比例的六水合硝酸钴和硝 酸铈溶于一定量的去离子水中, 摚拌时滴加过量的 双氧水, 溶液颜色逐渐从紫色变为橙黄色, 直至颜 色稳定。缓慢滴加氨水, 至悬浊液 $\mathrm{pH} \geqslant 10$ 。搅拌后 放置 $24 \mathrm{~h}$, 离心洗涤, 放入 $120^{\circ} \mathrm{C}$ 烘箱, $24 \mathrm{~h}$ 后取出 得到样品。采用该方法分别制备了钴掺杂比例为 0 、 $5 \mathrm{~mol} \% 、 15 \mathrm{~mol} \% 、 20 \mathrm{~mol} \% 、 25 \mathrm{~mol} \%$ 五种氧化铈粉 体, 以下均直接采用该 5 个百分数表示不同掺杂量 的氧化铈。

\section{3 催化剂的表征}

采用 $X$ 射线衍射(XRD D/max2500 型，日本日 立公司)来分析材料的物相和结构, 以 $\mathrm{Cu} \mathrm{K} \alpha$ 靶作为 辐射源，扫描速度 $2 \% \mathrm{~min}, 2 \theta$ 的扫描范围为 $20^{\circ} \sim$ $80^{\circ}$ 。

使用透射电子显微镜(TecnaiG220 型, 美国 FEI 公司)对材料微观结构进行观察和分析。使用 $\mathrm{X}$ 射线 光电子能谱仪 (XPS, ESCALAB 250Xi 型, 美国 Thermo Fisher 公司)对材料表面元素进行分析。使用 共聚焦激光拉曼仪(Raman, LabRAM HR Evolution 型, 法国 Horbia 公司)对材料内原子结构进行分析。 采用表面积及孔径分析仪(TristarII3020 型, 麦克默 瑞提克(上海)仪器有限公司)对材料的比表面积和孔 径分布进行分析。

\section{4 催化剂的电化学测试}

催化剂浆料的制备: 首先将等质量的催化剂与 乙炔黑混合研磨 $30 \mathrm{~min}$ 以上; 再称取 $5 \mathrm{mg}$ 混合物 粉末与 $5 \mu \mathrm{L} \mathrm{Nafion}$ 溶液和 $350 \mu \mathrm{L}$ 乙醇, 超声若干 分钟直至分散均匀; 最后, 用移液枪吸取 $7 \mu \mathrm{L}$ 催 化剂浆料滴在玻璃碳电极上, 玻璃碳上的催化剂的 负载量为 $0.40 \mathrm{mg} / \mathrm{cm}^{2}$ 。

采用 Autolab 电化学系统(PGSTAT302N 型, 瑞 士万通公司) 测量极化过程中的阻抗, 选择频率为 $0.01 \mathrm{~Hz} \sim 0.1 \mathrm{MHz}$, 电位为 $10 \mathrm{mV}$ 。采用电化学系统 (AFMSRX 旋转装置, AFCBP1 双恒电位仪, 美国 pine 公司), 利用旋转环盘电极(RRDE)技术来研究 催化剂的氧气还原和氧气析出催化活性。电极头由 玻璃碳圆盘电极 (直径 $5 \mathrm{~mm}$, 几何表面积为 $0.196 \mathrm{~cm}^{2}$ ) 和外围的铂环电极 (几何表面积为 $0.125 \mathrm{~cm}^{2}$ ) 构成。电化学测试在室温下进行, 采用标 准三电极体系进行测试, 铂丝为对电极, $\mathrm{Ag} / \mathrm{AgCl}$ 电 极(饱和 $\mathrm{KCl}$ 溶液)为参比电极, 装有玻璃碳电极头 (在玻璃碳表面覆盖有催化剂)的旋转盘电极(RDE) 为工作电极, 电解液为 $0.1 \mathrm{~mol} / \mathrm{L} \mathrm{KOH}$ 溶液, 由超纯 水(Millipore, $18.2 \mathrm{M} \Omega \cdot \mathrm{cm}$ )配置而成。电化学测试前, 
通高纯氮气超过 $30 \mathrm{~min}$, 排除电解质中的氧气。在 氮气饱和的 $0.1 \mathrm{~mol} / \mathrm{L} \mathrm{KOH}$ 溶液中测试玻璃碳上的 催化剂的电化学性能, 电势范围在-0.9 0.1 V(vs. $\mathrm{Ag} / \mathrm{AgCl})$, 扫速为 $50 \mathrm{mV} / \mathrm{s}$, 直至得到稳定的循环伏 安 $(C V)$ 曲线。然后再在 $-0.9 \sim 0.1 \mathrm{~V}$ 电势范围内以 $10 \mathrm{mV} / \mathrm{s}$ 的扫速, 测得 $\mathrm{CV}$ 曲线, 来确定非法拉第电 流 $\left(i_{\mathrm{Ar}-\mathrm{CV}}\right)$ 。

用 RRDE 研究催化剂对氧气还原的催化活性, 通入高纯氧气至少 $30 \mathrm{~min}$ 。在氧气饱和的 $0.1 \mathrm{~mol} / \mathrm{L}$ $\mathrm{KOH}$ 溶液中进行, 电势范围为 $-0.9 \sim 0.1 \mathrm{~V}$, 扫速为 $10 \mathrm{mV} / \mathrm{s}$, 电极转速分别为 $400 、 900 、 1600$ 和 $2500 \mathrm{r} / \mathrm{min}$ ，法拉第电流密度可以通过下面公式得到:

$$
j=\left(i_{\mathrm{ORR}}-i_{\mathrm{Ar}-\mathrm{CV}}\right) / S A_{\mathrm{geo}}
$$

其中 $i_{\mathrm{ORR}}$ 是 $\mathrm{ORR}$ 的电流, $S A_{\text {geo }}$ 是玻璃碳盘的几何面 积。根据 Koutecky-Levich 方程, ORR 动力学电流密 度满足以下公式:

$$
1 / j=1 / j_{\mathrm{k}}+1 / j_{\mathrm{d}}=1 / j_{\mathrm{k}}+1 /\left(B w^{1 / 2}\right)
$$

其中 $j$ 是测得的盘电流密度, $j_{\mathrm{k}}$ 和 $j_{\mathrm{d}}$ 分别对应动力学 电流密度和扩散极限电流密度, $w$ 是电极转速。而 $B$ 满足以下公式:

$$
B=0.62 n \mathrm{~F} D_{\mathrm{O}_{2}}{ }^{2 / 3} v^{-1 / 6} C_{\mathrm{O}_{2}}
$$

其中 $n$ 是 ORR 过程中的转移电子数, $\mathrm{F}$ 是法拉第常 数 $(96485 \mathrm{C} / \mathrm{mol}), D_{\mathrm{O}_{2}}$ 是 $\mathrm{O}_{2}$ 的扩散系数 $\left(D_{\mathrm{O}_{2}}=1.86 \times\right.$ $\left.10^{-5} \mathrm{~cm}^{2} / \mathrm{s}\right), v$ 是电解质的动力学粘度 $\left(v=0.01 \mathrm{~cm}^{2} / \mathrm{s}\right)$, $C_{\mathrm{O}_{2}}$ 是电解质中溶解 $\mathrm{O}_{2}$ 的浓度 $\left(C_{\mathrm{O}_{2}}=1.21 \times\right.$ $10^{-6} \mathrm{~mol} / \mathrm{cm}^{3}$ )。

在整个 RRDE 测试中, 环电位固定在 $0.5 \mathrm{~V}(v \mathrm{~s}$. $\mathrm{Ag} / \mathrm{AgCl}$ )用来氧化氧气还原过程中产生的 $\mathrm{H}_{2} \mathrm{O}_{2} /$ $\mathrm{HO}_{2}^{-}$。在碱性电解质中, 转移电子数 $n$ 可以用下面 的公式计算:

$$
n=4 \times \frac{I_{\mathrm{R}}}{I_{\mathrm{D}}+I_{\mathrm{R}} / \mathrm{N}}
$$

其中 $I_{\mathrm{D}}$ 是盘上的法拉第电流, $I_{\mathrm{R}}$ 是环上的法拉第电 流, $\mathrm{N}$ 是盘电极的收集系数 $(\mathrm{N}=0.22)$ 。

对于氧气析出反应测试, 电解质为氮气饱和的 $0.1 \mathrm{~mol} / \mathrm{L} \mathrm{KOH}$ 溶液, 工作电极的电位扫描范围为 $0 \sim 1.0 \mathrm{~V}(v s . \mathrm{Ag} / \mathrm{AgCl})$, 扫描速度为 $10 \mathrm{mV} / \mathrm{s}$, 电极转 速为 $1600 \mathrm{r} / \mathrm{min}$ 。最后将测出来的数据电位值加上 0.965 , 转换为相对可逆氢电极电位 (vs. RHE)。

\section{2 结果与讨论}

\section{1 掺杂量对催化剂结构和组成的影响}

图 1 所示为不同钴掺杂比例的氧化铈纳米粒子 的 XRD 图谱, 从图中可以看出, 制备的钴掺杂氧化
铈纳米粒子的主要衍射峰与 PDF 卡片(PDF\#780694)均吻合良好，因此可以判断所制备的氧化铈 晶体结构为立方晶系。但同时可以发现制备的材料 的结晶度较差, 在 $2 \theta=\sim 33^{\circ}$ 的氧化铈特征峰不明显, 推测是没有炦烧的结果。当摩尔掺杂比例为 $25 \mathrm{~mol} \%$ 时, 氧化铈的(111)、(200)和(311)三个主要晶面对应 的特征峰明显宽化, 表明钴掺杂比例过高会导致材 料晶格被严重破坏, 出现了大量无定型相, 因此氧 化铈的完整晶体结构已基本消失。

另外, 研究进一步发现随着钴掺杂量的增加, (111)晶面对应的特征峰强度有所减弱并出现宽化 现象，表明晶粒的尺寸也随之减小，催化性能则随 之提高。除此之外, 图 1(b)中, 氧化铈(111)面对应的 主峰随着钴掺杂量的增多有向低衍射角度移动的趋 势，这一现象表明钴进入了氧化铈晶格内部导致材 料氧空位增多，一定程度上提高了材料的催化能力。

图 2 所示分别为氧化铈纳米粒子的 $\mathrm{N}_{2}$ 吸附-脱 附曲线和 BJH 孔径分布曲线，其中氧化铈的比表面 积为 $105.84 \mathrm{~m}^{2} / \mathrm{g}$, 大小适中。根据图 2(a), 可以判断 吸附脱附曲线符合纳米粒子或用硬模板制备的大孔 材料的特征，相对压力超过 0.7 才出现滞后环，说明
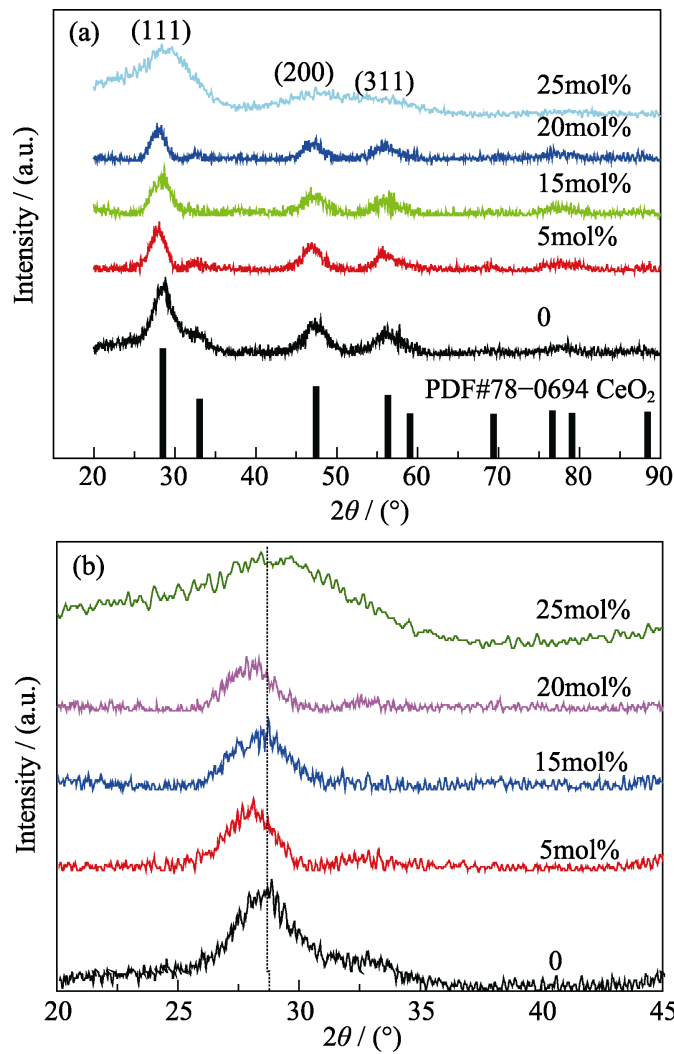

图 1 不同钴掺杂比例的氧化铈纳米粒子的 XRD 图谱

Fig. 1 XRD patterns of ceria nanoparticles with different doping ratio of cobalt

(a) Whole range patterns; (b) Magnified patterns of the highest peak (111) 

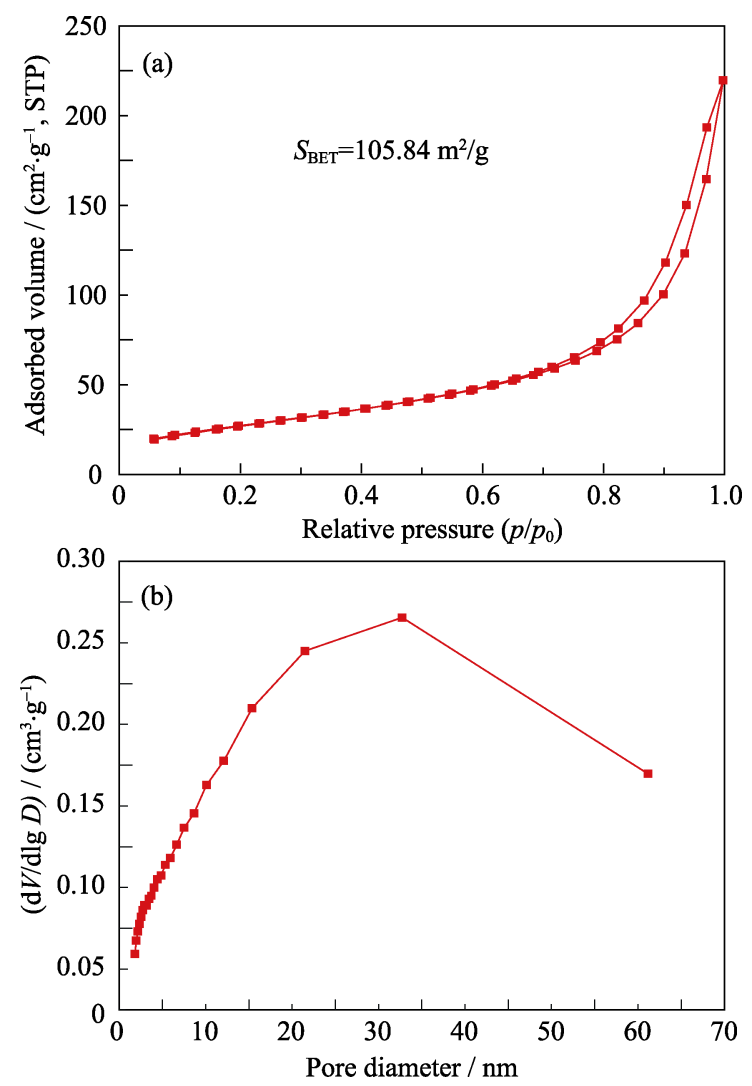

图 2 (a)氧化铈纳米粒子的 $\mathrm{N}_{2}$ 吸附-脱附曲线和(b)氧化铈 纳米粒子的 $\mathrm{BJH}$ 孔径分布曲线

Fig. 2 (a) $\mathrm{N}_{2}$ adsorption-desorption isotherm of ceria nanoparticles and (b) BJH pore size distribution curve of ceria nanoparticles

高压区吸附较快, 为 IV 类等温吸附曲线, 符合大孔 特点。此外，根据图 2(b)也可以推测制备的材料有 大量大孔。这些吸附曲线测试结果表明所制备的氧 化铈为纳米粒子。

\section{2 掺杂对电催化性能的影响}

研究发现掺杂少量的钴可以明显改变氧化铈的 催化性能。图 3 所示分别为不同掺杂比例的氧化铈 纳米粒子的 ORR 和 OER 极化曲线。由图 3(a)可以 看出，当掺杂摩尔比例为 $5 \mathrm{~mol} \%$ 时，氧化铈 ORR 的 LSV 曲线第一平台消失, 推测氧化铈在 ORR 中混 合电子转移过程有向 4 电子转移过程演变的趋势; 同时研究发现在不同钴掺杂量的氧化铈中，钴掺杂 量为 $20 \mathrm{~mol} \%$ 时氧化铈 ORR 的半波电位和起始电位 略低于其它掺杂量。总体上, ORR 的极限电流和起 始电位并没有明显改变, 表明钴的掺杂量并不显著 影响氧化铈对 ORR 的催化能力。由图 3(b) 可见, 随 着钴掺杂量的增加，极限电流密度也随之增大，但 掺杂量较多时, 其增幅并不显著。结合 XRD 图谱分 析, 表明钴掺杂比例为 $20 \mathrm{~mol} \%$ 的氧化铈为最优化 材料, 下面的工作主要以 $20 \mathrm{~mol} \%$ 钴掺杂量的氧化铈
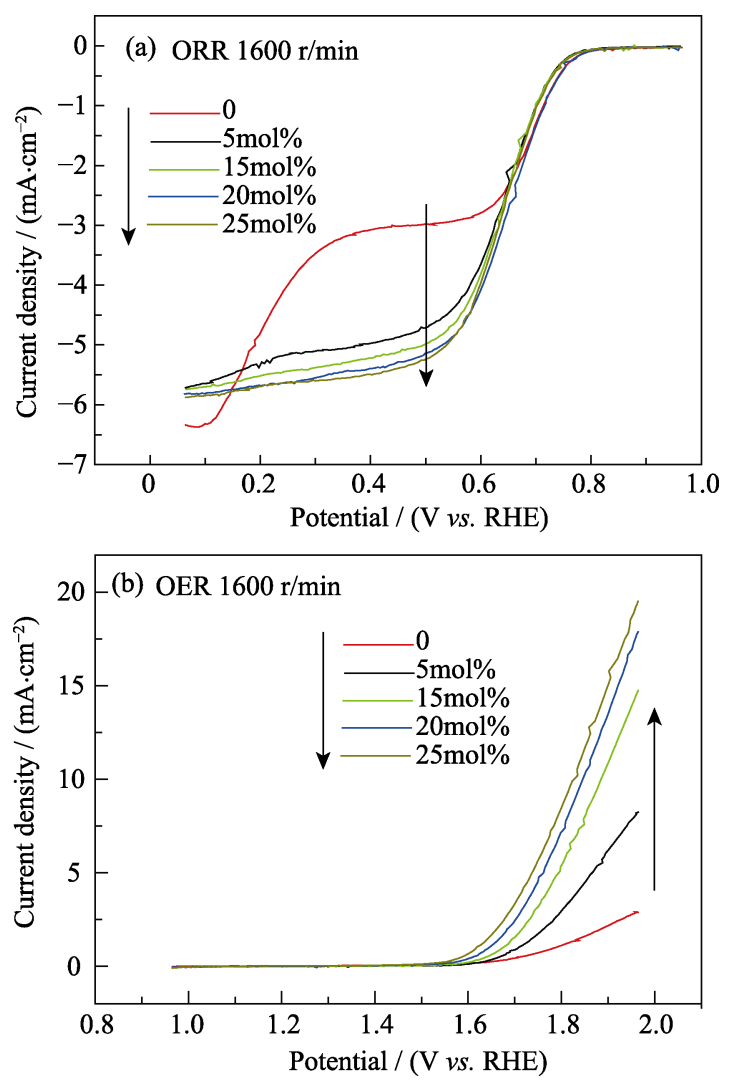

图 3 不同掺杂比例的氧化铈纳米粒子在 $1600 \mathrm{r} / \mathrm{min}$ 下的 ORR (a)和 OER (b)极化曲线

Fig. 3 Polarization curves at $1600 \mathrm{r} / \mathrm{min}$ of different of ceria nanoparticles with different doping ratio(a) (ORR) and (b) (OER)

和未掺杂钴的氧化铈进行对比, 深入讨论钴掺杂对 氧化铈电催化性能的影响。

由图 4(a)可见，掺杂后的氧化铈对 ORR 的催化 能力仍比商业化的 $\mathrm{Pt} / \mathrm{C}$ 稍差, 而在碱性或酸性条件 下很多碳材料 ${ }^{[9-11]}$ 、锰族简单氧化物 ${ }^{[12-14]}$ 以及尖晶 石类氧化物 ${ }^{[15-16]}$ 的电催化性能均可以和 $\mathrm{Pt} / \mathrm{C}$ 相妶美, 侧面表明了氧化铈本身并不适合用作电催化剂。另 外, 图 4(b)中, 其在碱性条件下的 OER 催化能力与 商业化的 $\mathrm{RuO}_{2}$ 差距不大, 表明钴掺杂后主要提升 的是氧化铈对 OER 的催化能力。根据碱性条件下 OER 催化机理 ${ }^{[17-18]}$ 分析, 这是掺杂后材料表面氧空 位增多 ${ }^{[8,19]}$ 、原子与晶格氧键能改变 ${ }^{[20]}$ 以及三价钴 离子较好的 OER 催化能力 ${ }^{[21-22]}$ 三方面协同作用的 结果。

由图 5(a)可以发现, 掺杂后的氧化铈转移电子 数为 3.8 , 符合 4 电子转移途径, 从 0.1 到 $0.7 \mathrm{~V}$ 转移 电子数相差不大; 而纯氧化铈在此范围内的转移 电子数却先上升, 下降后再上升, 由此可以推断氧 气还原反应在氧化铈上按二步来进行, 通过图 5(b) 中的 $\mathrm{K}-\mathrm{L}$ 曲线计算得到的转移电子数也证明了这 
一点。

通过图 6 所示的计时电流曲线可以发现, 氧化 铈在掺杂钴后, 在 OER 稳定性测试中要明显优于没
有掺杂钴的氧化铈, 并且在 ORR 稳定性测试中掺 杂钴的氧化铈稳定性也要好于未掺杂的氧化铈, 且 掺杂钴后氧化铈的稳定性均高于商业化的催化剂。
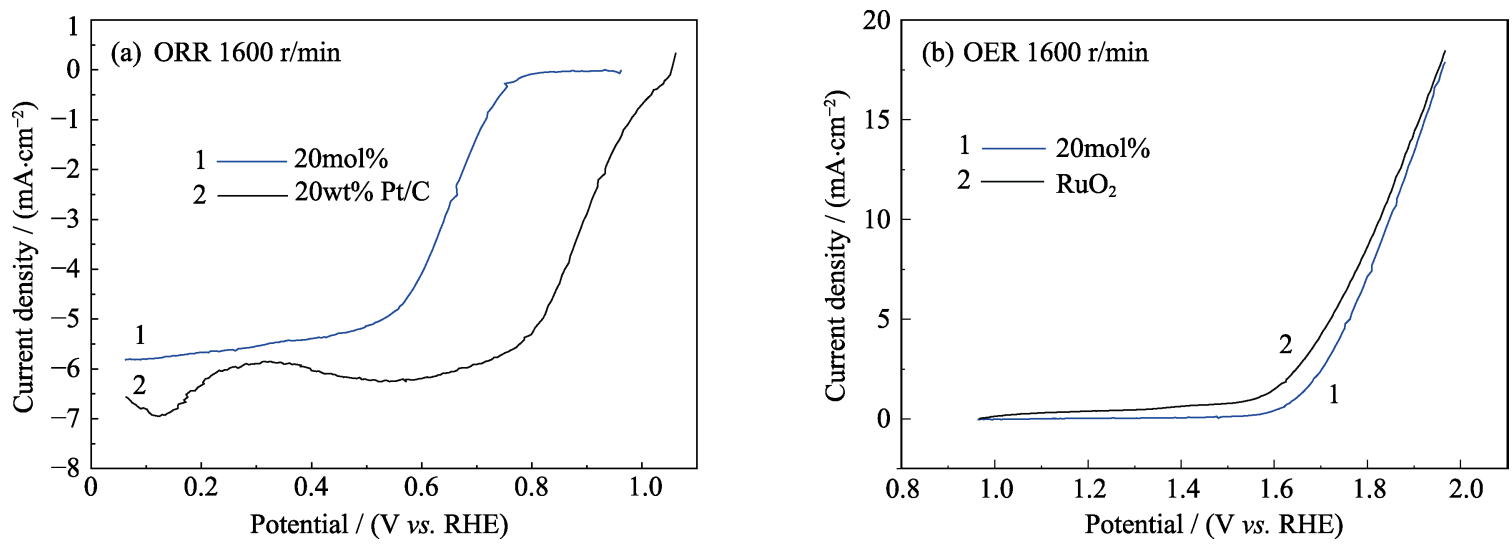

图 4 (a)氧化铈纳米粒子和 $\mathrm{Pt} / \mathrm{C}$ 在 $1600 \mathrm{r} / \mathrm{min}$ 下的极化曲线 $(\mathrm{ORR})$; (b)氧化铈纳米粒子和 $\mathrm{RuO}_{2}$ 在 $1600 \mathrm{r} / \mathrm{min}$ 下的极化曲线(OER)

Fig. 4 Polarization curves at $1600 \mathrm{r} / \mathrm{min}$ of (a) ceria nanoparticles and $\mathrm{Pt} / \mathrm{C}(\mathrm{ORR})$ and (b) ceria nanoparticles and $\mathrm{RuO} \mathrm{O}_{2}(\mathrm{OER})$
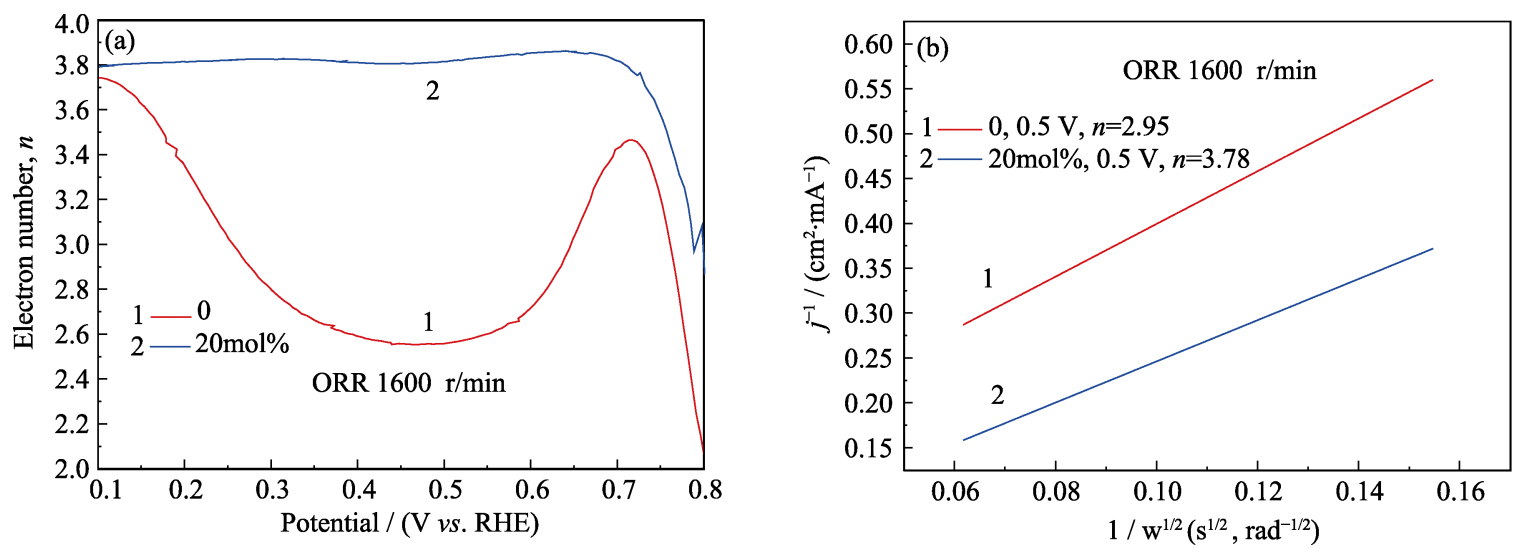

图 5 (a) RRDE 模式下测得相应掺杂比例的氧化铈纳米粒子转移电子数; (b) $0.5 \mathrm{~V}$ 时 相应掺杂比例的氧化铈纳米粒子的 K-L 曲线

Fig. 5 (a) Corresponding transfer electron number of ceria nanoparticles with different doping amount in RRDE model, and (b) corresponding K-L plots of ceria nanoparticles with different doping ratio at $0.5 \mathrm{~V}$
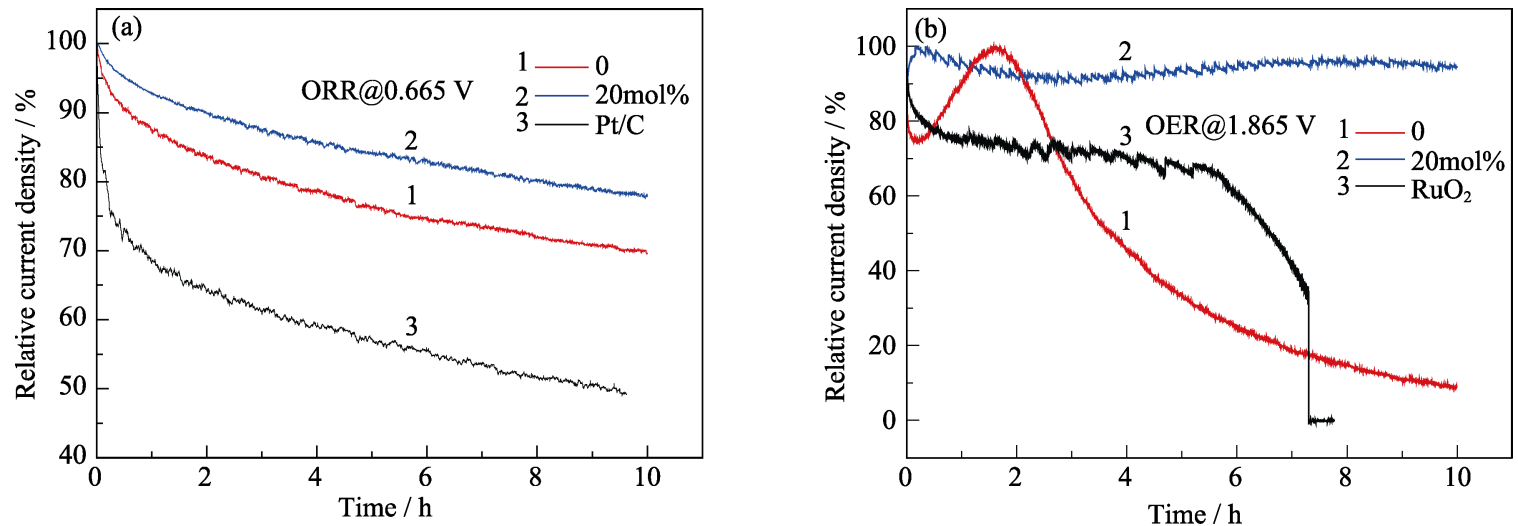

图 6 (a)二种不同掺杂比例的氧化铈纳米粒子和 $\mathrm{Pt} / \mathrm{C}$ 对应的计时电流曲线; (b)二种不同掺杂比例 氧化铈纳米粒子和 $\mathrm{RuO}_{2}$ 对应的计时电流曲线

Fig. 6 Current-time $(I-t)$ chronoamperometric responses for ceria nanoparticles (a) at two different doping ratio and $\mathrm{Pt} / \mathrm{C}$, and (b) at two different doping ratio and $\mathrm{RuO}_{2}$ 
参照文献[23-24], 得出了图 7(a)和(b)插图中的 等效电路, 其中 $R_{\mathrm{s}}$ 代表溶液阻抗、 $R_{\mathrm{f}}$ 代表催化剂膜 阻抗、 $R_{\mathrm{ct}}$ 代表电荷转移阻抗。结果显示在相同电压 下, ORR 过程中掺杂钴后的氧化铈电荷转移阻抗比 纯氧化铈要小。在 OER 阻抗测试中, 掺杂钴后的氧 化铈的电荷转移阻抗远大于催化剂膜阻抗, 该现象 应该与选择的测试电压有关; 同时测试表明, 掺杂 钴后的氧化铈的电荷转移阻抗数值要远低于纯氧 化铈, 这直接证明了掺杂钴后显著提高了氧化铈对 OER 的催化能力, 也从侧面证明了氧化铈导电性的 提高。

\subsection{Raman 光谱表征}

通过图 8 可以发现，氧化铈在 $455 \mathrm{~cm}^{-1}$ 左右位 置有一个很强的振动吸收峰, 对应氧化铈 F2g 对称 伸缩振动谱带。掺杂后, 此峰迁移到 $445 \mathrm{~cm}^{-1}$ 左右。 这应该是由于引入较小半径的 Co 离子后, 引起了 氧化铈晶格的扭曲变形, 致使该峰值发生红移。相 对于纯氧化铈的拉曼光谱图, 掺杂钴后的氧化铈拉 曼峰的强度明显降低, 这是由于氧化铈产生了更多 的缺陷 ${ }^{[8,25]}$ 。图谱在 $600 \mathrm{~cm}^{-1}$ 左右有一个宽峰, 一般 认为此峰与氧化铈的氧空位有关 ${ }^{[25-26]}$ 。同时谱图
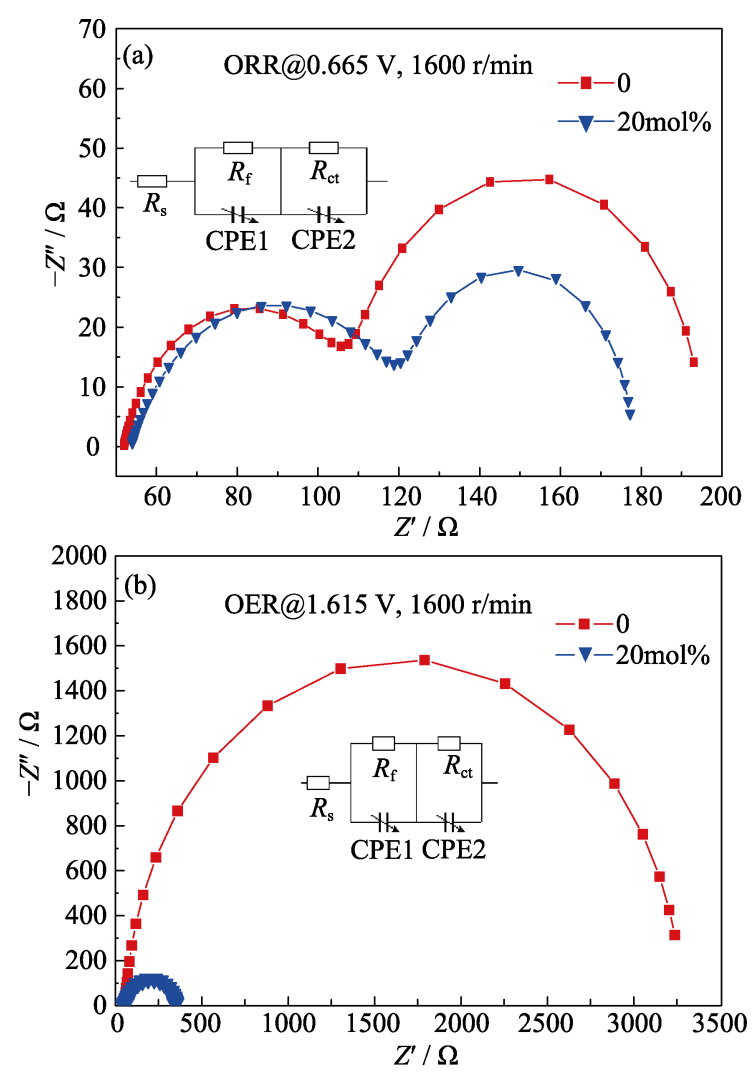

图 7 (a)二种不同掺杂比例下对应氧化铈的 ORR (a)和 OER (b)的极化阻抗图谱

Fig. 7 (a) Corresponding ORR(a) and OER (b) electrochemical impedance spectra of Ceria at two different doping ratio

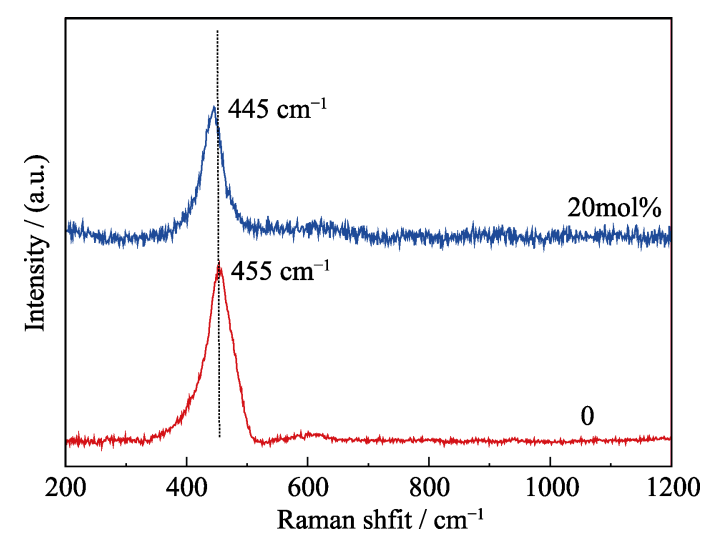

图 8 二种不同钴掺杂比例下对应氧化铈的拉曼光谱图

Fig. 8 Corresponding Raman spectra of Ceria at two different doping ratio of $\mathrm{Co}$

表明, 在钴掺杂比例为 $20 \mathrm{~mol} \%$ 时氧化铈的该峰面 积与 $450 \mathrm{~cm}^{-1}$ 左右的峰面积比值明显高于纯氧化铈, 说明钴掺杂增加了氧空位的数量, 有助于增强材料 的电催化能力。

\section{4 掺杂对催化剂内部晶体结构的影响}

图 9 分别为纯氧化铈和钴掺杂比例为 $20 \mathrm{~mol} \%$ 的氧化铈的透射电子显微镜和高分辨透射电子显微 镜照片, 从中可以直接观察到纳米粒子的直径为 $\sim 5 \mathrm{~nm}$, 但团聚情况比较严重。研究发现纳米粒子大 都暴露低指数的(111)晶面, 说明在没有表面活性剂 等外在因素下形成的样品大多趋近于最稳定的状 态。通过高倍透射电子显微镜分析可以发现, 在掺 杂后的氧化铈 HRTEM 照片(图 9(d))中没有看到图 9(b)蓝色线圈中高度有序的晶格条纹带, 从掺杂后 的氧化铈电镜照片中看到有少量条纹带断裂、错乱 (图 9(d)中红色线框), 并且晶格条纹间距变大, 这些 都是材料缺陷增多的具体表现, 也在一定程度上说 明了钴掺杂有利于提高氧化铈的催化活性。

\subsection{XPS 表征}

图 10 为不同钴掺杂比例氧化铈的XPS 图谱, 为 进一步研究掺杂对氧化铈内部结构的影响情况, 将 XPS 分峰拟合后得到的结果进行了列表分析(表 1)。

根据相关文献 ${ }^{[27-30]}$, 其中 $\mathrm{Ce}^{4+}=u^{\prime \prime \prime}+u^{\prime \prime}+u+$ $v^{\prime \prime \prime}+v^{\prime \prime}+v, \mathrm{Ce}^{3+}=u^{\prime}+v^{\prime}$ 。图 10 中 $\mathrm{O}_{\alpha}, \mathrm{O}_{\beta}, \mathrm{O}_{\delta}$ 和 $\mathrm{O}_{\gamma}$ 分别代表晶格氧、 $\mathrm{O}^{2-} / \mathrm{O}^{-}$、吸附的 $-\mathrm{OH}$ 和 $\mathrm{O}_{2}$ 、表面

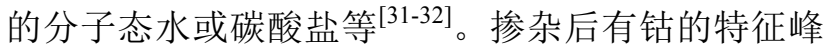
出现, 但不明显, 说明 Co 的含量较少。根据钴的 XPS 图谱可见, 钴还是以二价态为主, 但三价态的 钴可能在 OER 过程中起到主要催化作用 ${ }^{[22,33-34]}$ 。根 据 Ce3d XPS 谱图, 可以发现钴掺杂后 Ce 3d 特征峰 有向高结合能移动的趋势, 并且强度明显减弱, 这 

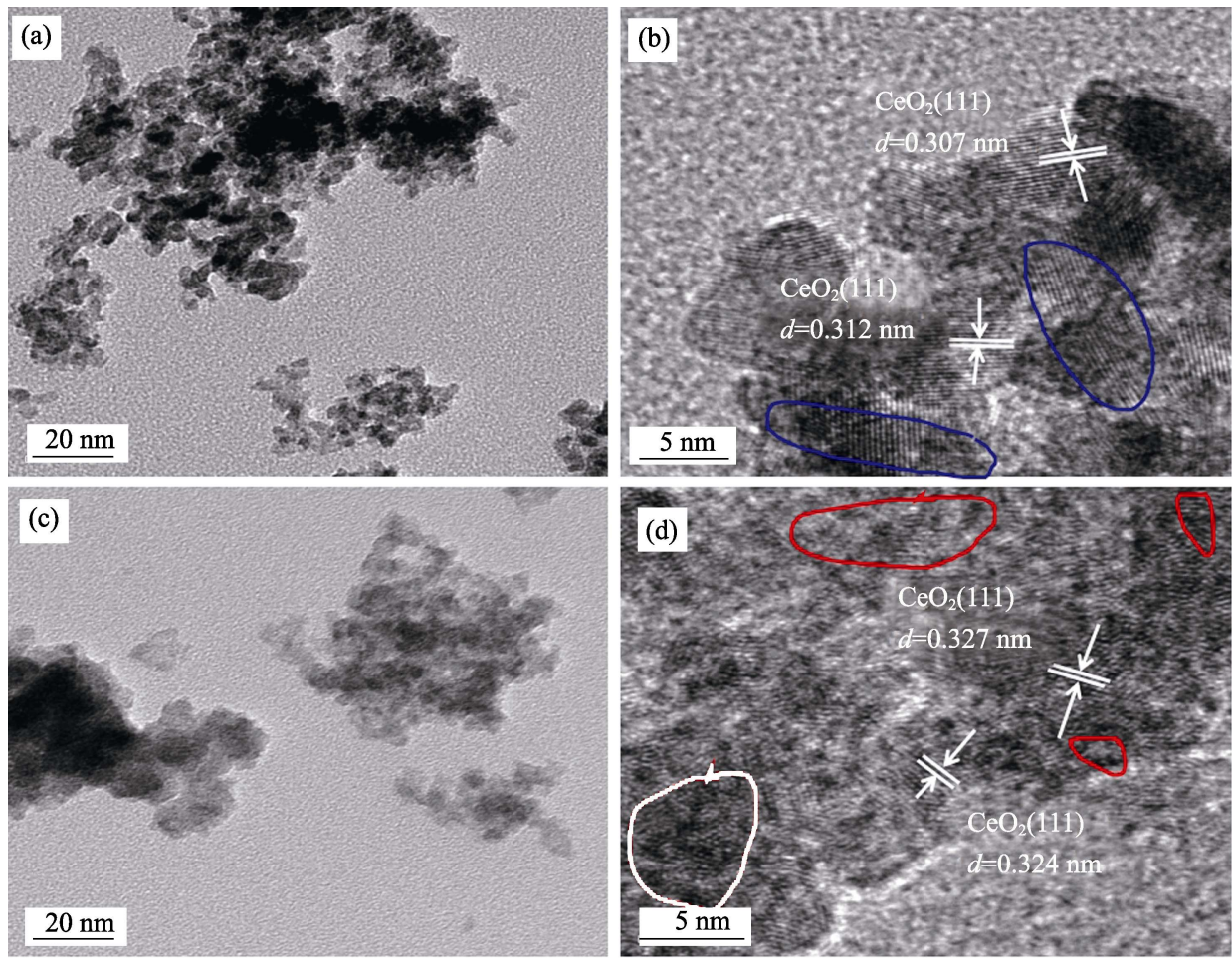

图 9 二种不同掺杂比例下对应氧化铈的 TEM 和 HRTEM 照片

Fig. 9 Corresponding TEM and HRTEM images of Ceria at two different doping ratio (a, b) $0 ;(\mathrm{c}, \mathrm{d}) 20 \mathrm{~mol} \%$
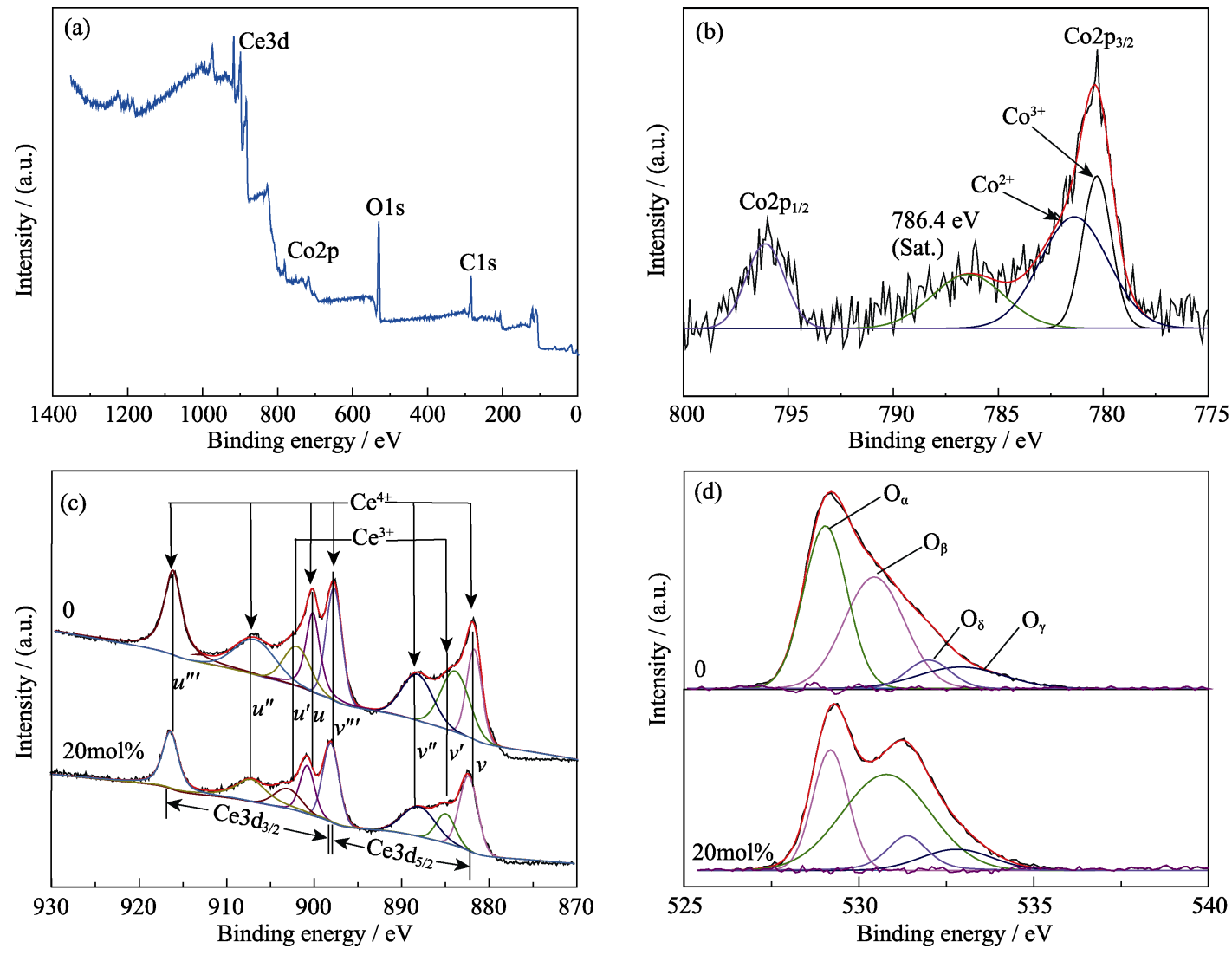

图 $10 \mathrm{Co}$ 掺杂比例为 $20 \mathrm{~mol} \%$ 时 $\mathrm{CeO}_{2}$ 的(a) XPS 全谱和(b) $\mathrm{Co} 2 \mathrm{p}$ 的高分辨 XPS 图谱; 不同掺杂比例下 $\mathrm{CeO}_{2}$ 中 $\mathrm{Ce} 3 \mathrm{~d}(\mathrm{c})$ 和 $\mathrm{O} 1 \mathrm{~s}(\mathrm{~d})$ 的高分辨 XPS 图谱

Fig. 10 (a) XPS survey spectra and (b) high-resolution XPS spectra of $\mathrm{Co}_{2} \mathrm{p}$ of $\mathrm{CeO}_{2}$ doping with $20 \mathrm{~mol} \% \mathrm{Co}$; High-resolution XPS spectra of $\mathrm{Ce} 3 \mathrm{~d}(\mathrm{c})$ and $\mathrm{O} 1 \mathrm{~s}(\mathrm{~d})$ of $\mathrm{CeO}_{2}$ with different doping ratio 
表 1 不同掺杂比例下对应的 XPS 结果

Table 1 Corresponding XPS results of different doping ratio

\begin{tabular}{ccccccc}
\hline \multirow{2}{*}{ Sample } & \multicolumn{6}{c}{ Content } \\
\cline { 2 - 7 } & $\mathrm{O}_{\alpha} /$ at $\%$ & $\mathrm{O}_{\beta} / \mathrm{at} \%$ & $\mathrm{O}_{\delta} / \mathrm{at} \%$ & $\mathrm{O}_{\gamma} / \mathrm{at} \%$ & $\mathrm{O}_{\beta} / \mathrm{O}_{\alpha}$ & $\begin{array}{c}{\left[\mathrm{Ce}^{3+} /\left(\mathrm{Ce}^{4+}\right.\right.} \\
\left.\left.+\mathrm{Ce}^{3+}\right)\right] / \%\end{array}$ \\
\hline 0 & 48.5 & 29.2 & 10.4 & 11.8 & 0.60 & 20.1 \\
$20 \mathrm{~mol} \%$ & 27.2 & 53.1 & 10.5 & 9.2 & 1.95 & 24.1 \\
\hline
\end{tabular}

些都说明钴掺杂起到了催化作用。同时, 分析表 1 可以看出, $\mathrm{O}_{\beta}$ 占总体 $\mathrm{O}$ 含量的百分比在掺杂后明显 增大, 而相关文献 $[32,35]$ 也能够证实 $\mathrm{O}_{\beta}$ 的增加有 利于催化性能的提高。另外, 表中 $\mathrm{Ce}^{3+}$ 含量的增加 也说明氧空位数量增多, 有利于催化反应的进行。

\section{3 结论}

\section{采用均相沉淀法制备了掺杂钴的氧化铈纳米粒} 子, 电化学测试表明掺杂摩尔比例为 $20 \mathrm{~mol} \%$ 时催 化效果最好, 且在长达 $10 \mathrm{~h}$ 的催化活性测试过程中, 该纳米粒子对 ORR 和 OER 均表现出了良好的催化 稳定性; XPS 谱图分析表明, 晶格氧的含量在掺杂 后由 48.5\%降低到 27.2\%; EIS 测试分析表明，掺杂 后测得的 OER 电荷转移阻抗数值要远低于未掺杂 时的相应值。钴掺杂后提高了电子导电性, 增加了 氧化铈的氧空位以及表面氧活性物种, 这三个方面 是改变氧化铈电催化性能的主要因素。研究结果为 其它离子导体材料用作金属-空气电池阴极催化剂 提供了思路。

\section{参考文献:}

[1] JIE S, KEVIN J M, HUBERT A G, et al. A perovskite oxide optimized for oxygen evolution catalysis from molecular orbital principles. Science, 2011, 334(6061): 1383-1385.

[2] JIN S, HUBERT A G, YABUUCHI N, et al. Design principles for oxygen-reduction activity on perovskite oxide catalysts for fuel cells and metal-air batteries. Nature Chemistry, 2011, 3(8): 647.

[3] WU G, KARREN L M, JOHNSTON C M, et al. High-performance electrocatalysts for oxygen reduction derived from polyaniline, iron, and cobalt. Science, 2011, 332(6028): 443-447.

[4] LEFÈVRE M, PROIETTI E, JAOUEN F, et al. Iron-based catalysts with improved oxygen reduction activity in polymer electrolyte fuel cells. Science, 2009, 324(5923): 71-74.

[5] CAO Z X, DING Y M, WANG Z C, et al. Porous calcium manganese oxide: preparation and electrocatalytic activity of oxygen reduction reaction. Journal of Inorganic Materials, 2017, 32(5): 535-542.

[6] HOU F, LI H, YANG Y, et al. Preparation and catalytic oxidation of $\mathrm{CO}$ with specific morphology and porous nano $\mathrm{CeO}_{2}$. Chemical Industry and Engineering Progress, 2017, 36(7): 2481-2487.

[7] KE J, XIAO J W, ZHU W, et al. Dopant-induced modification of active site structure and surface bonding mode for high-performance nanocatalysts: $\mathrm{CO}$ oxidation on capping-free(110)-oriented $\mathrm{CeO}_{2}$ : $\mathrm{Ln}(\mathrm{Ln}=\mathrm{La}-\mathrm{Lu})$ nanowires. Journal of the American Chemical Society, 2013, 135(40): 15191-15200.

[8] XU B, ZHANG Q, YUAN S, et al. Synthesis and photocatalytic performance of yttrium-doped $\mathrm{CeO}_{2}$ with a porous broom-like hierarchical structure. Applied Catalysis B: Environmental, 2016, 183: 361-370.

[9] HAO L, YU J, XU X, et al. Nitrogen-doped $\mathrm{MoS}_{2} /$ carbon as highly oxygen-permeable and stable catalysts for oxygen reduction reaction in microbial fuel cells. Journal of Power Sources, 2017, 339: 68-79.

[10] CHEN P, ZHOU T, XING L, et al. Atomically dispersed iron-nitrogen species as electrocatalysts for bifunctional oxygen evolution and reduction reactions. Angewandte Chemie International Edition, 2017, 56(2): 610-614.

[11] ZHU Q L, XIA W, AKITA T, et al. Metal-organic framework-derived honeycomb-like open porous nanostructures as precious-metal-free catalysts for highly efficient oxygen electroreduction. Advanced Materials, 2016, 28(30): 6391-6398.

[12] FENG J, LIANG Y G, WANG H L, et al. Engineering manganese oxide/nanocarbon hybrid materials for oxygen reduction electrocatalysis. Nano Research, 2012, 5(10): 718-725.

[13] TAN Y M, XU C F, CHEN G X, et al. Facile synthesis of manganese-oxide-containing mesoporous nitrogen-doped carbon for efficient oxygen reduction. Advanced Functional Materials, 2012, 22(21): 4584-4591.

[14] DONG C, LIU Z W, LIU Y J, et al. Modest oxygen-defective amorphous manganese-based nanoparticle mullite with superior overall electrocatalytic performance for oxygen reduction reaction. Small, 2017, 13(16): 1603903-1-9.

[15] LIANG Y Y, WANG H L, ZHOU J G, et al. Covalent hybrid of spinel manganese-cobalt oxide and graphene as advanced oxygen reduction electrocatalysts. Journal of the American Chemical Society, 2012, 134(7): 3517-3523.

[16] PENDASHTEH A, PALAMA J, ANDERSON M, et al. Ni$\mathrm{CoMnO}_{4}$ nanoparticles on N-doped graphene: highly efficient bifunctional electrocatalyst for oxygen reduction/evolution reactions. Applied Catalysis B: Environmental, 2017, 201: 241-252.

[17] HARDIN W G, MEFFORD J T, SLANAC D A, et al. Tuning the electrocatalytic activity of perovskites through active site variation and support interactions. Chemistry of Materials, 2014, 26(11): 3368-3376.

[18] HARDIN W G, SLANAC D A, WANG X, et al. Highly active, nonprecious metal perovskite electrocatalysts for bifunctional metal-air battery electrodes. The Journal of Physical Chemistry Letters, 2013, 4(8): 1254-1259.

[19] HARTMANN P, BREZESINSKI T, SANN J, et al. Defect chemistry of oxide nanomaterials with high surface area: ordered mesoporous thin films of the oxygen storage catalyst $\mathrm{CeO}_{2}-\mathrm{ZrO}_{2}$. ACS Nano. 2013, 7(4): 2999-3013.

[20] XU X M, SU C, ZHOU W, et al. Co-doping strategy for developing perovskite oxides as highly efficient electrocatalysts for oxygen evolution reaction. Advanced Science, 2016, 3(2): 1500187$1-6$.

[21] ZHU Y L, ZHOU W, SUNARSO J, et al. Phosphorus-doped perovskite oxide as highly efficient water oxidation electrocatalyst in alkaline solution. Advanced Functional Materials, 2016, 26(32): 5862-5872.

[22] HE Y, ZHANG J F, HE G W, et al. Ultrathin $\mathrm{Co}_{3} \mathrm{O}_{4}$ nanofilm as an efficient bifunctional catalyst for oxygen evolution and reduction reaction in rechargeable zinc-air batteries. Nanoscale, 2017, 9(25): 
8623-8630.

[23] MENG Y T, SONG W Q, HUANG H, et al. Structure-property relationship of bifunctional $\mathrm{MnO}_{2}$ nanostructures: highly efficient, ultra-stable electrochemical water oxidation and oxygen reduction reaction catalysts identified in alkaline media. Journal of the American Chemical Society, 2014, 136(32): 11452-11464.

[24] ZHAO X, FU Y, WANG J, et al. Ni-doped $\mathrm{CoFe}_{2} \mathrm{O}_{4}$ hollow nanospheres as efficient bi-functional catalysts. Electrochimica Acta, 2016, 201: 172-178.

[25] XU B, ZHANG Q T, YUAN S S, et al. Synthesis and photocatalytic performance of yttrium-doped $\mathrm{CeO}_{2}$ with a hollow sphere structure. Catalysis Today, 2017, 281: 135-143.

[26] LI H, MENG F, GONG J, et al. Structural, morphological and optical properties of shuttle-like $\mathrm{CeO}_{2}$ synthesized by a facile hydrothermal method. Journal of Alloys and Compounds, 2017, 722: 489-498.

[27] DENG W, DAI Q G, LAO Y J, et al. Low temperature catalytic combustion of 1, 2-dichlorobenzene over $\mathrm{CeO}_{2}-\mathrm{TiO}_{2}$ mixed oxide catalysts. Applied Catalysis B: Environmental, 2016, 181: 848-861.

[28] ZANG C J, ZHANG X S, HU S Y, et al. The role of exposed facets in the Fenton-like reactivity of $\mathrm{CeO}_{2}$ nanocrystal to the Orange II. Applied Catalysis B: Environmental, 2017, 216: 106-113.

[29] FENG J, YE S H, XU H, et al. Design and synthesis of FeOOH/
$\mathrm{CeO}_{2}$ heterolayered nanotube electrocatalysts for the oxygen evolution reaction. Advanced Materials, 2016, 28(23): 4698-4703.

[30] BECHE E, CHARVIN P, PERARNAU D, et al. Ce $3 \mathrm{~d}$ XPS investigation of cerium oxides and mixed cerium oxide $\left(\mathrm{Ce}_{x} \mathrm{Ti}_{y} \mathrm{O}_{z}\right)$. Surface and Interface Analysis, 2008, 40(3/4): 264-267.

[31] LI L L, ZHANG L, MA K L, et al. Ultra-low loading of copper modified $\mathrm{TiO}_{2} / \mathrm{CeO}_{2}$ catalysts for low-temperature selective catalytic reduction of $\mathrm{NO}$ by $\mathrm{NH}_{3}$. Applied Catalysis B: Environmental, 2017, 207: 366-375.

[32] LIANG F L, YU Y, ZHOU W, et al. Highly defective $\mathrm{CeO}_{2}$ as a promoter for efficient and stable water oxidation. Journal of Material Chemistry, 2015, 3(2): 634-640.

[33] XU L, JIANG Q Q, XIAO Z H, et al. Plasma-engraved $\mathrm{Co}_{3} \mathrm{O}_{4}$ nanosheets with oxygen vacancies and high surface area for the oxygen evolution reaction. Angewandte Chemie International Edition, 2016, 55(17): 5277-5281.

[34] ZHUANG L Z, GE L, YANG Y S, et al. Ultrathin iron-cobalt oxide nanosheets with abundant oxygen vacancies for the oxygen evolution reaction. Advanced Materials, 2017, 29(17): 1606793$1-7$.

[35] WANG F, ZHANG L, XU L, et al. Low temperature CO oxidation and $\mathrm{CH}_{4}$ combustion over $\mathrm{Co}_{3} \mathrm{O}_{4}$ nanosheets. Fuel, 2017, 203: 419-429. 